\title{
Integration of practice nurse competencies in general practices in Belgium: a qualitative evaluation study of a postgraduate program.
}

Evi Matthys ( $\square$ Evi.matthys@uantwerpen.be)

Universiteit Antwerpen https://orcid.org/0000-0002-1269-5714

Roy Remmen

Universiteit Antwerpen

Peter Van Bogaert

Universiteit Antwerpen

Research article

Keywords: Practice nurses, primary healthcare, education, general practitioners, postgraduate course, multidisciplinary collaboration, shared care, evaluation

Posted Date: September 12th, 2019

DOI: https://doi.org/10.21203/rs.2.14413/v1

License: (c) (i) This work is licensed under a Creative Commons Attribution 4.0 International License. Read Full License 


\section{Abstract}

Background Recently, first steps were taken to educate primary care nurses in Belgium and consequently trained practice nurses have only recently been introduced in Belgian general practices. Therefore, it is unclear what the experiences of general practitioners (GPs) and trained practice nurses are concerning these new multidisciplinary collaborations. With this study we would like to report new insights in the visions and experiences of GPs and practice nurses concerning the new postgraduate education for practice nurses, the current and future roles and responsibilities taken on by practice nurses, and the experienced collaboration between GPs and practice nurses.

Methods A qualitative research methodology was chosen, based on the principles of a descriptive qualitative research design. This study was conducted in primary healthcare in Belgium. Four focus group interviews were conducted. A total of twenty practice nurses and ten GPs participated. The practice nurses had followed or were currently following the postgraduate program for practice nurses, all the GPs were experienced in collaborating with a trained practice nurse.

Results Four major themes could be derived from the data: practice nurses' roles and responsibilities, importance of protocol care, consequences of the collaboration, and future opportunities and perspectives. GPs expressed the importance of education for practice nurses, in order for them to take on more responsibilities in the practices. Trained practice nurses showed more initiative towards taking on a broader range of tasks. They both expressed the importance of care protocols in support of a good multidisciplinary collaboration. The impact on the daily practice was experienced as far-reaching by GPs. While collaborating with trained practice nurses, GPs experienced the ability to provide a higher quality of care.

Conclusion Both GPs and trained practice nurses have positive experiences about providing shared care in general practices. GPs were surprised by the initiative, shown by practice nurses, and by the broad range of tasks nurses took on. The impact of this collaboration on both the daily practice and the quality of patient care, is unanimously experienced as positive, even though it entails a higher workload. GPs have specific future expectations about the practice nurse education and roles.

\section{Background}

An ageing population with more chronic diseases and multi-morbidities, combined with reduced hospital stays, is challenging primary health care systems around the world (1). An increase in the number of chronic patients and multi-morbidity patients leads to more complex care needs $(2,3)$. In addition, in Europe, patients become more demanding and expect health care to be accessible and of high quality at the same time, while health professionals experience increasingly high workloads, leading to an increased outflow from the profession (4-6).

It is expected that primary health care systems are able to support the entire organization of health care. This in order for the entire organization to become more responsive to the changing health needs, to offer 
a more integrated care and to increase the efficiency of provision of health care (7). To best meet these more complex care needs, new models of shared-care based on multidisciplinary practice are being developed, in order to provide a high-quality person-centered care. In addition, the current roles of health professionals are being modernized $(8,9)$.

In Belgium, single-handed practices of general practitioners (GPs) have been the main form of primary care provision for years. Recently a trend is emerging towards introducing more group practices where multiple primary care physicians collaborate $(9,10)$. This, in an attempt to address the higher workload caused by the shortened/reduced hospital stays and the increased needs for preventive and chronic care at primary care level (2). In addition, there is an evolution in the interprofessional support in the practices. Primary care physicians are now collaborating with other health professionals such as primary care nurses, psychologists, social workers, etc (11). As a consequence of the increasing collaboration in primary care, new health professional roles are evolving, including those among the nursing workforce (12).

Primarily, nurses were introduced in primary care practices to substitute for a number of tasks, and therefore, in order to meet a perceived shortage of primary care physicians (13). Over time, to continue to guarantee quality care, nursing roles and responsibilities have expanded. Their area of primary care expertise increased and nurses started taking on tasks concerning prevention, education and chronic care besides performing technical nursing skills and taking on administrative tasks. Due to the shift in primary care needs, there is a rise in educational programs to train nurses to obtain the required skills and competencies (14). Many countries are in the process of reforming nursing education and have moved the primary nursing education fully or partially to Bachelor levels (14). In Belgium, the postgraduate course 'Nursing in Primary Care Practices' was created and is offered as a supplement to the Bachelor program which provides no specific primary care training or internships in primary care practices (15).

Currently, practice nurses primarily play a role in chronic disease management, prevention advise, patient education, medication management and administration. Only a limited number of practice nurses participates in primary care policy making and research (16). There's a high variety in nursing roles across the world, according to the context and local needs. Findings concerning the impact of primary care nursing, on the other hand, are much more similar around the world. Interprofessional collaboration between GPs and practice nurses seems to have a positive effect on patient satisfaction, hospital admission and mortality (13).

Recently, first steps were taken to educate primary care nurses in Belgium and consequently specifically trained practice nurses have just recently been integrated in Belgian general practices. Therefore, it is unclear what the experiences of general practitioners and trained practice nurses are concerning these new multidisciplinary collaborations, which tasks practice nurses can take on in the practices and what the visions of general practitioners and practice nurses are about future developments towards interprofessional collaboration. 
After performing a quantitative study where we assessed GP attitudes towards practice nurse integration and task suitability of practice nurses in their practices, we proposed a follow-up study to find out more about the experiences of GPs and practice nurses themselves (11). The results of the survey showed the majority of GPs to have positive attitudes towards collaboration with practice nurses. However the job profile and ethical framework of practice nurses remained insufficiently clear. Nurses were found most suitable to take on tasks concerning patient education and technical nursing skills, and least suitable to develop evidence based protocols and to perform administrative tasks (11).

With this qualitative study we would like to report new insights in the visions and experiences of GPs and practice nurses concerning the new postgraduate education for practice nurses, the current and future roles and responsibilities taken on by trained practice nurses, and the experienced collaboration between GPs and trained practice nurses.

\section{Methods}

\section{Aim}

With this qualitative research we aim to answer the following research questions:

- How do practice nurses and GPs experience the current postgraduate education of practice nurses? And to what extent does it support the collaboration between GPs and practice nurses?

- How do trained practice nurses and GPs experience this new collaboration where tasks and responsibilities are taken on by trained practice nurses?

- What are the opinions of GPs and practice nurses on future opportunities for shared care in primary care practices?

\section{Qualitative approach and research paradigm}

A qualitative research methodology was chosen. The recent nature of the employment of nurses in a general practice, required an exploratory approach, based on the principles of descriptive qualitative research design (17). The approach was based on a constructivist research paradigm, where the researchers find knowledge to be context and temporally dependent. The respondents were motivated to share their personal opinions on the research topic. Respondents and interviewer were both influenced by contextual factors, for example, the current context of our health care system. Therefore, this interaction resulted in influenced results.

\section{Researcher characteristics and reflexivity}

Two researchers conducted the focus group interviews with practice nurses. The interviewer was a graduate student in nursing and midwifery sciences and has a bachelor of science in nursing with 
thorough knowledge of the competencies of nursing. The secretary (first author) was an experienced home care nurse, and practice nurse with understanding of the organizational needs of general practices. Both researchers had no former relationship with the nurses.

Two researchers conducted the focus group interview with GPs. This time, the secretary (first author) of the interviews with practice nurses, took on the role as interviewer. The secretary (second author) was an experienced nurse and researcher, with a specific interest in primary care nursing.

\section{Context and Sampling strategy}

This study was conducted in primary healthcare in Belgium, including general practice care. Nurses with experience in primary healthcare and GPs with experience in multidisciplinary collaboration in their practices were recruited for this study. We applied a purposive sampling strategy. During the sampling the researchers focused on including respondents with a variety in work experience, educational background, age and type of general practice (single handed/group practice). Possible participants were recruited through the postgraduate course for practice nurses at the University of Antwerp.

The postgraduate course is founded by the center for general medicine (ELIZA department) and the department of nursing and midwifery sciences at the University of Antwerp (Belgium). This initiative is in collaboration with University colleges Artesis Plantijn, Karel de Grote and Thomas More, and is supported by the province of Antwerp and the city of Antwerp. This 1-year course was created and launched in 2016 (15). The main goal of this course is introducing practice nurses in general practices, where nurses support GPs while treating, supporting and caring for a patient population with complex care needs. This entails the introduction of practice nurses with specific clinical and organizational competencies and skills, so they are able to care for patients with a wide range of health questions and/or multi morbidity of biological, psychological, social and existential nature. The nurses are actively and autonomously deployed in practices where they perform nursing, clinical and organizational tasks in collaboration with general practitioners. The course entails four modules that are based on the learning objectives. The modules are: practice organization and communication; health, prevention and patient education; target specific protocol care, care planning, case management and integrated care; clinical practice (see Table 1). The clinical practice entails 240 hours of internship in a general practice combined with the development and implementation of an evidence based quality improvement project. The graduation project challenges students to realize a real interprofessional care provision, with the focus on the experiences of patients and patient outcomes.

The postgraduate course aims for an integrated employment of practice nurses whereby a real interprofessional collaboration between practice nurses and GPs is established. Interprofessional collaboration is defined as a collaboration where a multiprofessional team develops a common vision and a goal-oriented approach, through dialogue and with a shared responsibility. This collaboration is characterized by interaction between the various professions, where there is an exchange of information, ideas and recommendations through frequent consultations (18). 
Students of the postgraduate course were informed and asked to participate. It was emphasized that participation was entirely without obligation and that the research was in no way related to the postgraduate course or able to influence the course. This way we were able to include sixteen nurses. In addition, practice nurses were also recruited by contacting general practices by email and phone. Another 4 nurses could be included. GPs were also included through the postgraduate course for practice nurses, since these GPs opened their practices as internship locations for practice nurses. This way, ten GPs could be included. They all were experienced in collaborating with practice nurses, half of them only through internships, and the other half through internships and permanent employment of practice nurses.

\section{Ethical issues pertaining to human subject}

See section 'Ethics approval and consent to participate' under the heading 'Declarations'.

\section{Data collection methods, instruments and technologies}

Four focus group interviews were conducted. Three with practice nurses, one with GPs. Attendance at each focus group interview for nurses ranged from four to ten nurses. The dates of the focus group interviews were: $1 / 2 / 2018,28 / 3 / 2018$ and $29 / 3 / 2018$. The interviews lasted respectively 60,80 and 80 minutes. Ten GPs attended the GP focus group interview. This interview took place on 29/6/2018 and lasted 90 minutes. Table 2 provides more information on the composition and heterogeneity of these focus groups. The interviews were all recorded.

Table 2 should appear at this location

At the beginning of each focus group interview, nurses and GPs were asked to fill out a questionnaire to collect some socio-demographic information (nurses: sex, age, currently following the postgraduate course, currently working, duration of work experience and other care providers in the practice) (GPs: sex, age, years of experience as a GP, support in the practice, area and financing of the practice). After the first focus group session with nurses, the socio-demographic questionnaire was adapted and three questions were added (working hours per week, area of the practice and financing system of the practice). The secretary wrote memos, concerning non-verbal communication, during all the interviews, and the researchers also wrote memos when familiarizing with the data. An interview guide was used during the focus group sessions, this guide was also adapted after the data-analysis of the first focus group with nurses. The researchers noticed some questions were rather irrelevant. The response on other questions was remarkably in-depth causing the researchers to expand the focus on certain topics such as responsibilities of nurses in a new multiprofessional collaboration. Information obtained during the first focus group was re-suggested by the interviewer during the second and third interview, in order to find out if more information or other points of view would come up. The questions in the interview guide for nurses were based on searching the literature, previous research and discussion among the researchers. 
The questions in the interview guide for GPs were based on the information gained from the focus group interviews with practice nurses, on searching the literature and on discussion among the researchers. Table 3 presents an overview of the different topics that were discussed.

\section{Data processing and analysis}

The recorded data were transcribed in a Word file. This file was uploaded and analyzed in the software package NVivo 12. The memos were entered manually into NVivo.

After each focus group interview, two researchers independently transcribed the recordings within 48 hours, and after familiarizing with the data. The researchers listened to the interview once more, before starting with the transcription. In a second stage, the researchers independently divided the text fragments and added descriptive codes. Then, interpretative codes were given, with the research question kept in mind. During the analyses, recurring, remarkable opinions of the respondents were identified as relevant themes to answer the research question.

\section{Techniques to enhance trustworthiness}

During the interviews, there was enough time for a thorough look into the opinions of the respondents. The interviewer asked in-depth questions regarding meaning and context, in order to enhance the richness of the data. The two researchers performed the data analyses independently, resulting in a codebook which was made up in consensus.

The data analysis was a spiral, iterative process with systematic repetition of the discussed stages and application of reflexivity. After the initial coding, a second coding took place later on. And during the process of coding, a constant check with the former interviews took place, in order to guard the original meaning (19).

The researchers were constantly aware of their own ideas or presuppositions that could possibly influence the research. To increase the neutrality of the researchers, possible interactions and considerations were recorded in numerous memos. Self-reflection, captured in memos, was promoting for the consistency of the research. The voice of the respondents was heard clearly by the use of literal quotes, and they firmly supported the findings of the researchers.

Every stage of the research process (protocol-reporting) is objectively documented, enhancing the transparency of this research.

In order to meet the standards for reporting qualitative research, reports were made according to the 'Standards for Reporting Qualitative Research' (SRQR) checklist (20).

\section{Results}


A total of $10 \mathrm{GPs}$ and 20 PNs agreed to participate in the focus group interviews. All the GPs reported to have experience with collaborating with PNs through the nurses' internships. Half of the GPs had experience with a multidisciplinary collaboration through a permanent PN employment in the practice. All the PNs were experienced in collaborating with GPs, all of them through internships, eight of them also through a permanent employment as a PN. Table 2 presents the socio-demographic and work(place) characteristics of the participants.

Four major themes could be derived from the data: practice nurses' roles and responsibilities, the importance of protocol care, consequences of the (interprofessional) collaboration, and future opportunities and perspectives.

\section{Theme 1: Practice nurses' roles and responsibilities}

\section{a) Taking on tasks in the practice}

During their education, nurses were trained to obtain competencies across four domains (Table 1). Practice nurses themselves declared to perform a wide variety of tasks within the practices, overlapping the three domains, including the clinical practice domain (internship). Practice organization and communication; Health, prevention and patient education; Target specific protocol care, care planning and case management.

Practice nurses often started off in practices taking on tasks related to reception and administration, this in order to gradually learn more about the daily functioning of the practice and the patient population. At the beginning of their internships, practice nurses declared GPs to have limited knowledge of the nurses' expertise in a practice. Consequently, GPs declared to be surprised by the large amount of tasks practice nurses took on in their practices.

"In the beginning I started off with some administrative work, learned to know the patient files and all that, it was very easy. And then, gradually, I took on more technical nursing tasks, and now I also take on chronic care in the practice." (PN, Female (F), 32 years old (yrs.))

"It was the first time we had a PN in our practice, so it was a bit of a search in the beginning. We mainly engaged her in chronic care and prevention, the follow up of chronic patients in particular. She more than fulfilled our expectations there." (GP, Male (M), 66 yrs.)

Within weeks, after taking on administrative tasks and performing technical nursing skills (eg; drawing blood, taking blood pressures, ...), most of the practice nurses started taking on the follow up of chronic care patients. This by organizing nursing consults, according to an evidence based protocol, for patients with a specific chronic condition. Often patients with diabetes or kidney insufficiency or chronic obstructive pulmonary disease. In addition nurses took on tasks related to organization of extramural care. 
"My tasks vary, in the beginning I mainly took on blood sampling, then I followed the postgraduate course, and learned how to perform spirometry tests, so now I perform those as well. Uihm... I also organize multidisciplinary team meetings, vaccinations, I also inform patients about vaccinations, I perform simple wound care, I freeze warts, I take electrocardiograms. I conduct intake-interviews for new patients in our practice, and I go on house calls." (PN, F, 50 yrs.)

"We were already highly supported in our administration, so very soon we asked the PN to follow up a diabetes patient for example. It is so pleasant to be able to tell patients to come back for a follow up by the $P N . "$ (GP, M, 62 yrs.)

"Last year, I developed a project around sexually transmitted disease (STD) screening. Now I take on the STD screening for patients who are not experiencing any symptoms, so really a preventive screening." (PN, F, 33 yrs.)

\section{b) Importance of education}

GPs expressed the importance of education for practice nurses, in order to take on more responsibilities in the practice.

The roles of practice nurses without specific education were often restricted to taking on administrative tasks and to performing technical nursing skills. Whereas the roles of practice nurses with post graduate education were more extensive. This was often caused by the nurses, who initiated taking on a broader range of tasks and therefore more responsibilities. GPs declared practice nurse trainees to take on tasks more independently, with more confidence. In comparison, GPs noticed practice nurses without specific education to be more executive employees, who took fewer initiatives and whose roles were limited to defined tasks, delegated by the GPs.

"I really felt like a secretary, I did not see any patients, so I just did administrative work. And during the postgraduate course, we learned about the follow-up of patients with diabetes, and I took the initiative to confront the GP with the fact that I wanted to do more in the practice. After that, I started taking on diabetes care in the practice." (PN, F, 50 yrs.)

"That's a benefit of the postgraduate education, we received a document that stated which tasks a nurse can take on in a practice. So I showed it to the GPs and they were very surprised by all the nursing tasks we could perform. They still had such a dated image of us, nurses." (PN, F, 41 yrs.)

"... with the added value of being able to hand over more tasks to PNs who completed the postgraduate course. For instance even individual consultations that occur simultaneously with GP consultations, or a more in-depth follow up, where we (GPS) tend to fall short by a lack of time." (GP, $F, 31$ yrs.)

"Our (employed) PN takes on more executive tasks, clear, well-defined. And she does that very well, but it needs to be well-defined. Whereas the educated nurse, she was able to take on the entire preventive policy 


\section{Theme 2: The importance of protocol care}

Both GPs an practice nurses expressed the importance of care protocols in support of a good multidisciplinary collaboration. GPs felt more confident handing over tasks they have always been carrying out. Practice nurses also felt more confident taking on these tasks, when the care process and consults were molded in a fixed structure.

Additionally, GPs noticed when tasks were properly delineated, by being described in a protocol, they were taken on easily by practice nurses, even after a short period of time. However, when there were only verbal agreements concerning shared care, GPs declared nurses being less venturous in taking on tasks. For both GPs and practice nurses it often remained insufficiently clear what the former agreements were, since there was no written document to fall back on.

"There's a protocol for the frail elderly patients in our practice. We schedule our monthly home visits with these patients, and that runs very smoothly. GPs simply trust us and we alert them if there are any problems or we instantly schedule a GP home visit." (PN, F, 50 yrs.)

"In our practice, it was important that everything was written down. Everything that was put down in writing and communicated, not only with the PN, but also with each other (GPS), that went well. But all the other tasks, only partly written out or only verbally communicated, just did not happen, if the protocol wasn't there." (GP, $M, 50$ yrs.)

Concerning the development of these protocols, it was less clear and unambiguous who should take on this responsibility. Practice nurses declared to experience difficulties in taking on this tasks, mainly because they felt too inexperienced and therefore insecure to bear that responsibility.

"We have two trained PNs in our practice, and indeed, protocols are necessary, but it takes time to implement them. And to evaluate them, to get an idea of how patients are experiencing care, and if they experience an improvement. Goal oriented care is just as important for me. To know what the individual goals of your patients are and to work towards those goals. Then, protocols fall into the background, by being fully embedded in the daily practice, they become invisible." (GP, F, 42 yrs.)

"I do feel like I fall short in developing protocols myself. For example, I wanted to take on a chronic care consult. I have a lot of ideas about how I want to do this, but in order to translate these ideas into an evidence based protocol, I do need help from a GP." (PN, F, 33 yrs.)

\section{Theme 3: Consequences of the (interprofessional) collaboration}




\section{a) Patient level}

Both GPs and PNs noticed positive effects of their collaboration on patient care. GPs noticed PNs to take a position in the practice that was missing before. Such a degree of accessibility/approachability was formerly never present in a general practice. Because of this, patients were surprisingly open towards PNs, often sharing relevant information that previously would not have been shared with the GPs. By collaborating interprofessionally, both GPs and PNs had access to more information, allowing the GP to pick up and elaborate on the information obtained through the PNs.

"We tend to state that we as GPs are approachable for patients, but those nurses are way more approachable and therefore they (PNs) get a whole different perspective on the patients. Because they interact with them differently. Our knowledge of the patients' context increases and consequently we can approach them with more regard of their context." (GP, F, 42 yrs.)

"Patients are more open towards PNs, on different levels. For instance, because you are more approachable as a nurse, you learn more about their underlying problems. Maybe that's because they feel you make more time for them, or communicate with them differently. But at the same time, when they have been waiting a long time in the waiting room, you get the complaints, and minutes later when they enter the GPS consultation room, suddenly it's all good. (PN, F, 31 yrs.)

It surprised me, like, I expected a bigger struggle/hurdle for them (patients). But it depends on how the GP introduces you. If they display an air of trust, patients pick up on that. (PN, F, 34 yrs.)

\section{b) Daily practice}

The impact on the daily practice was experienced as far-reaching by GPs. Employment of (or providing an internship for) PNs translated into more work, into an increased need for consultation moments between the different professions, but also into a higher job satisfaction for GPs. While collaborating with practice nurses, GPs experienced the ability to provide a higher quality of care, caused by the expertise of PNs and the extra time that could be reserved for the patients during the consultations by the nurses.

"Also more meetings, where you normally just act upon your own ideas, now you coordinate them with the PN. When you have a different background and education, your conceptual frameworks also differ. Putting ideas together in order to solve the puzzle. That's definitely a limitation of working more independently." (GP, F, 31 yrs.)

"It's an improvement for the quality of care. They (PNs) take on tasks that we could no longer prioritize, and were feeling a bit guilty about. For instance, our complex chronic care patients, we should make more time for them. Now, with the PN, we discuss these patients. That is such an enrichment, but as any quality improvement, it increases our workload." (GP, M, 62 yrs.) 


\section{Theme 4: Future opportunities and perspectives}

\section{a) PNs' roles}

GPs experienced changes in the local context of their practices and in the patient needs, this due to socio demographic evolutions. GPs expressed the necessity of introducing more professional caregivers in their practices, and the chances for PNs to expand their roles in the practices. While there is an evolution towards larger group practices and multidisciplinary practices, GPs experienced the organization of these practices to become a fulltime job. Opinions differed across GPs on the extent to which PNs could take on more tasks regarding the management and coordination of these practices. According to some GPs, PNs could coordinate the daily functioning of these practices, allowing the GPs to regain focus on their core business. Other GPs disagreed and were more cautious about the growth of their practices and the extent to which they would hand over control of the management of the practices.

"In the future, I see the PN role more in the center of the practice. If she has the qualifications, I would not mind at all to hand over the coordination and leadership and say 'you make sure the practice runs smoothly'. Compared to where we are now, that's a leap forward, but we will have to, or at least, I would find it a good evolution if that would be possible within primary care." (GP, M, 62 yrs.)

"I don't think you can manage such a system financially, I'm just saying, at one point you will have an organization with a PN who only makes sure everything else is working." (GP, M, 60 yrs.)

GPs did agree unanimously on the added value of a structure that allows PNs to share knowledge and experiences among each other, beyond the boundaries of the individual general practices. Similar to the local quality groups that exist for Belgian GPs.

"And exchange information, they (PNs) need to come together and hear from one another what they are doing, what is going well, what isn't." (GP, F, 42 yrs.)

\section{b) Education}

Both GPs an PNs had certain future expectations about the education program of PNs. GPs stated that they expected the postgraduate course to help streamline the employment in general.

PNs themselves noticed a lack of practice opportunities in specific nursing skills. Particularly those skills that occur more in general practice settings compared to hospital settings, were insufficiently addressed during the course. This resulted in PNs feeling insecure while performing those skills in the practices. In addition, PNs shared the desire to be guided by an employed PN during their internships, in comparison to the current internship guidance by GPs. 
"I hope, with a specific education like this, a few general agreements will be made. Everyone is doing the same work at this point, protocols are being made in every single practice. How could we provide more structure in general, so the work in the individual practices is facilitated?" (GP, F, 31 yrs.)

"For me, the postgraduate education did not entail enough practice opportunities. For instance, taking a smear and those kind of tasks. Now we learn how to do that in the practice, but I don't think that's appropriate to perform that task for the first time during an internship. I don't know, the course lacked something like a skills lab." (PN, F, 56 yrs.)

GPs additionally expressed the growing need for an expansion of their own education, since human resource competencies become more important while general practices keep on growing. PNs also noticed opportunities for an expansion of the education of GPs. According to PNs, their education should be focused more on the role of PNs. This in order for graduating GPs to have a better understanding of the expertise and competencies of trained PNs.

"It wouldn't be such a bad idea if GPs get the same training we got." (PN, F, 56 yrs.)

\section{Discussion}

This study provides a more in-depth view on the visions and experiences of trained practice nurses and GPs about practice nurse education and integration in Belgian general practices.

\section{Interprofessional collaboration and education}

Both GPs and practice nurses declared the limited awareness of GPs concerning the nurses' expertise and competencies. This limited knowledge impacts the degree of an actual interprofessional collaboration (21). When GPs are more aware of the nurses' expertise and competencies, it allows them to employ and collaborate with practice nurses in a more integrated manner. A superficial knowledge of the nurses' expertise often leads to a more instrumental collaboration where practice nurses perform a limited range of tasks, administrative tasks and taking on technical nursing skills, delegated by the GPs (22). This knowledge deficit of GPs is founded in the absence of interprofessional education for Belgian GPs and practice nurses. According to the World Health Organization, interprofessional education is essential to the development of a collaborative practice-ready health workforce (23). Students have improved perceptions of professions that will potentially be members of their future practice teams, after they have had the opportunity to learn alongside students from those other professions (24). Belgian GPs only experience the presence of other healthcare professionals at that point where they enter the work field, and this could possibly restrict an integrated interprofessional collaboration with other professionals. In addition, PN are only recently being introduced in Belgian general practices. Therefore, opportunities of getting to know them as members of an interprofessional health care team are rather scarce. 


\section{Importance of postgraduate education}

The range of tasks, taken on by practice nurses in the practices, gradually increased during their internships. At first practice nurses got to know the practices, the daily routine, their colleagues and the patient population. After a few weeks, practice nurses started taking on more elaborate tasks like providing chronic care, prevention and promotion advise, in collaboration with the GPs. It is remarkable that even practice nurses who were already employed as practice nurses before they started the postgraduate course, saw new opportunities to take on tasks in their own practices. Even GPs noticed remarkable differences between practice nurses who did or did not follow the training. This suggests that education does not only teach nurses which tasks can be taken on by them in a general practice, but it also provides nurses the necessary support to feel confident enough to initiate taking on these tasks. A positive association between postgraduate education at any level and more time spent in advanced practice activities confirms this reasoning (25). In addition, evidence confirms the direct and indirect benefits of successful learning transfer through changes in attitudes, application of knowledge, improved critical thinking and decision-making skills, confidence, ability to challenge treatment decisions, and increased job satisfaction (26-28). Furthermore, the relationship between educational preparation and patient outcomes is characterized by a decreased patient mortality and reduced failure to rescue when there are more nurses with higher levels of education (29).

\section{Importance of protocol care}

Initiating collaborative care provision appeared to be supported by the presence of (evidence-based) protocols. Both GPs and practice nurses declared to feel more confident to initiate collaborating while providing care when these care processes were molded into fixed structures. On the other hand, once GPs and nurses are used to the new collaboration, it was stressed by GPs that it is important for protocols to fall into the background. At that point they become part of the daily practice, but are no longer visibly present. This to be able to monitor that care provision remains sufficiently patient-oriented, tailored to individual needs. In general, standardized care provision is critically examined (30). Evidence-based care should admit that it tends toward standardization of clinical decisions, which will reduce individual discretion for both caregivers and patients. On the other hand, non-standardized care promotes individual discretion, and therefore might also lead to greater variability in clinical care (30). Care provision is challenged to find common ground between the two approaches, allowing the provided care to be sufficiently standardized, without excluding an individual approach (31).

\section{Development of evidence based protocols}

Where GPs and practice nurses both agreed on the importance of protocol care, it appeared to be less clear who should be responsible for the development of these protocols. Belgian GPs already indicated to consider practice nurses less suitable to develop these protocols (11). Practice nurses themselves 
declared to feel insecure to take on that task, mainly because they felt insufficiently educated in that area. So far, this has led to GP trainees taking on the task of writing these protocols. It is rather peculiar for one profession to be singularly responsible for the development of the blueprint of a care that is provided by multiple professions. Stakeholder involvement is crucial in the development and implementation of protocols and guidelines (32). Practice nurses should be educated in this area so they feel sufficiently confident to take on this task, in collaboration with GPs and other stakeholders. This also raises the question where patients should be situated with regard to the development of these primary care protocols. Their involvement might lead to a more individualized patient care, without precluding the evidence base (33).

\section{Barriers for interprofessional collaboration}

Insufficient financial support and the lack of a legal framework for practice nurses hinders Belgian GPs to initiate collaborations with practice nurses (11). During the interviews, these barriers were briefly mentioned by GPs and practice nurses. The researchers however did not elaborate on this, since these are known and well-described barriers within the Belgian primary care context (34). Worldwide, governments are taking initiatives to support the employment of practice nurses. But, governmental support can only be effective and truly supportive when linked to a number of requirements that create the conditions for practice nurses to work within their area of expertise (35). Besides these known barriers, this qualitative research discovered the lack-of-knowledge-barrier. GPs who have insufficient knowledge of the expertise and skills of practice nurses, possibly will not fully integrate them in their practices, even with government support.

\section{Limitations}

PNs were included through the postgraduate course for PNs at the University of Antwerp. Here, the question can be asked to what extent an internship experience differs from a real work experience. Eight of the included PNs however, were already employed as PNs at the start of the training. Above all, it was important for the researchers to look at experiences of PNs who were specifically trained to take on tasks in the general practice setting. Similarly, researchers wanted to gain insight in experiences of GPs who work together with specifically trained practice nurses. Innovative practices are at the center of this research. Those practices are taking the initiative to collaborate with trained practice nurses, despite existing barriers.

Another limitation is the singular focus group interview with GPs. One or more additional focus group interviews might provide a more in-depth view on their visions and experiences. And it would confirm data-saturation. The limited number of GPs who are already collaborating with a trained practice nurse, has mainly determined that only one interview took place. 


\section{Future research}

Due to changing care needs, the organization of primary care in Belgium continues to evolve. Future research is necessary to find out how the education of practice nurses can also respond to these changes. Additional research may also be useful to find out how the training of general practitioners can better meet the complex needs of the current primary care organization. In addition, future research is recommended to determine which measures GPs can support more in the process of integrating practice nurses into their general practices.

\section{Conclusion}

Both GPs and PNs have positive experiences about providing shared care in general practices. Nurses positively experience the ability to take on a broad range of nursing tasks, where they are specifically trained for. GPs are surprised by the initiative, shown by PNs, to take on tasks in the practices. The impact of this collaboration on both the daily practice and the quality of patient care, is unanimously experienced as positive, even though it entails a higher workload. GPs express different opinions on future roles of PNs in the general practices. In the light of future evolutions within primary care, both GPs and PNs have specific expectations towards the current education of PNs, and towards the education of GPs.

\section{Abbreviations}

\section{F-Female}

FG-Focus Group

GP(s)-General practitioner(s)

M-Male

PN(s)-Practice nurse(s)

SRQR-Standards for Reporting Qualitative Research

STD-Sexually Transmitted Disease

Yrs-Years old

\section{Declarations}

\section{Ethics approval and consent to participate}

Before the start of the study, we obtained approval from the ethics committee of Antwerp University Hospital (see additional file 1). During sampling, respondents were informed that their participation would 
be entirely voluntary and that it would be possible to end their participation at any time. Participants were informed that the entire conversation would be recorded, but that their privacy would be guaranteed from the data analysis on by pseudonymisation. Each respondent received an information letter and signed an informed consent at the start of the interview. The respondents were once again informed that ending their participation was possible at any time during the interview.

\section{Consent for publication}

Each respondent received an information letter and signed an informed consent at the start of the interview. In the paragraph 'confidentiality of data' the researchers explained the consequences of participating regarding publication of data.

\section{Availability of data and materials}

The datasets generated and analysed during the current study are available in the Figshare repository, https://doi.org/10.6084/m9.figshare.9777911.v1.

\section{Competing interests}

The authors declare that they have no competing interests. The authors alone are responsible for the content and writing of this article. 'Wit-Gele Kruis van Antwerpen vzw' has no role in the design of the study and collection, analysis, and interpretation of data in writing the manuscript.

\section{Funding}

The first author, Evi Matthys, received a scholarship from the home care organization 'Wit-Gele Kruis van Antwerpen vzw', to perform a part time PhD.

\section{Authors' contributions}

EM designed the topic list, organized the focus group interviews, transcribed and analyzed the data, and wrote the manuscript. PVB and RR contributed in writing the manuscript. All authors read and approved the final manuscript.

\section{Acknowledgements}

We thank Ms Merve Imemek for her support as interviewer during the focus group interviews, and her support during data transcription and data analysis. Also, a special thanks goes out to the home care 
organization 'Wit-Gele Kruis van Antwerpen vzw', for its financial support.

\section{Additional files}

Additional file 1:.pdf-Approval ethics committee-Additional file 1 provides the approval from the ethics committee of Antwerp University Hospital to perform this research.

\section{References}

1.World Health Organization (WHO). Global strategy and action plan on ageing and health (2016-2020). 2016 http://www.who.int/ageing/global-strategy/en/. Accessed 12 June 2018.

2.Araujo de Carvalho I, Epping-Jordan J, Pot AM, Kelley E, Toro N, Thiyagarajan JA, et al. Organizing integrated health-care services to meet older people's needs. Bulletin of the World Health Organization. 2017;95(11):756-63.

3.Osborn R, Moulds D, Schneider EC, Doty MM, Squires D, Sarnak DO. Primary Care Physicians In Ten Countries Report Challenges Caring For Patients With Complex Health Needs. Health affairs (Project Hope). 2015;34(12):2104-12.

4.Goetz K, Musselmann B, Szecsenyi J, Joos S. The influence of workload and health behavior on job satisfaction of general practitioners. Family medicine. 2013;45(2):95-101.

5.Kacenelenbogen N, Offermans AM, Roland M. Burnout of general practitioners in Belgium: societal consequences and paths to solutions. Revue medicale de Bruxelles. 2011;32(4):413-23.

6.Lorant V, Geert C, Hoore W,. Huisartsgeneeskunde: aantrekkingskracht en beroepstrouw bevorderen. https://kce.fgov.be/nl/publication/report/huisartsgeneeskunde-aantrekkingskracht-en-beroepstrouwbevorderen: Federaal Kenniscentrum voor de Gezondheidszorg (KCE); 2008. Accessed 7 July 2018.

7.Kringos DS, Boerma WGW, Hutchinson A, Saltman RB. Building primary care in a changing Europe. Copenhagen (Denmark): World Health Organization; 2015.

8.Nolte E, Knai, C. \& Saltman, R. B. (eds). Assessing chronic disease management in European health systems: concepts and approaches (2014). United Kingdom: World Health Organization; 2014.

9.OECD/EU. Health at a Glance: Europe 2016: OECD Publishing; 2016. http://dx.doi.org/10.1787/9789264265592-en.

10.Jabaaij L, Hingstman L. Alleen is maar alleen: huisartsen steeds vaker samen. Huisarts \& Wetenschap 2007;50(5). 
11. Matthys E, Remmen R, Van Bogaert P. Practice nurse support and task suitability in a general practice: a cross-sectional survey in Belgium. Journal of Interprofessional Care. 2019:1-9.

12.Delamaire M, Lafortune G. Nurses in Advanced Roles: A Description and Evaluation of Experiences in 12 Developed Countries2010; (54):[106 p.]. http://dx.doi.org/10.1787/5kmbrcfms5g7-en.

13.Martinez-Gonzalez NA, Djalali S, Tandjung R, Huber-Geismann F, Markun S, Wensing M, et al. Substitution of physicians by nurses in primary care: a systematic review and meta-analysis. BMC health services research. 2014;14:214.

14.Lahtinen P, Leino-Kilpi H, Salminen L. Nursing education in the European higher education area variations in implementation. Nurse education today. 2014;34(6):1040-7.

15.Antwerp University. Nursing in a general practice 2018.

https://www.uantwerpen.be/en/education/education-and-training/nursing-general-practice/profile/. Accessed on 28 July 2018.

16.Norful A, Martsolf G, de Jacq K, Poghosyan L. Utilization of registered nurses in primary care teams: A systematic review. International journal of nursing studies. 2017;74:15-23.

17.Colorafi KJ, Evans B. Qualitative Descriptive Methods in Health Science Research. Herd. 2016;9(4):1625.

18.Choi BC, Pak AW. Multidisciplinarity, interdisciplinarity and transdisciplinarity in health research, services, education and policy: 1. Definitions, objectives, and evidence of effectiveness. Clinical and investigative medicine Medecine clinique et experimentale. 2006;29(6):351-64.

19.Pope C, Ziebland S, Mays N. Qualitative research in health care. Analysing qualitative data. BMJ (Clinical research ed). 2000;320(7227):114-6.

20.0'Brien BC, Harris IB, Beckman TJ, Reed DA, Cook DA. Standards for reporting qualitative research: a synthesis of recommendations. Academic medicine: journal of the Association of American Medical Colleges. 2014;89(9):1245-51.

21.Green BN, Johnson CD. Interprofessional collaboration in research, education, and clinical practice: working together for a better future. The Journal of chiropractic education. 2015;29(1):1-10.

22.D’Amour D, Ferrada-Videla M, San Martin Rodriguez L, Beaulieu M-D. The conceptual basis for interprofessional collaboration: Core concepts and theoretical frameworks. Journal of Interprofessional Care. 2005;19(sup1):116-31.

23.World Health Organization (WHO). Framework for action on interprofessional education and collaborative practice. Switzerland (Geneva): WHO; 2010. 
24.Ateah CA, Snow W, Wener P, MacDonald L, Metge C, Davis P, et al. Stereotyping as a barrier to collaboration: Does interprofessional education make a difference? Nurse education today. 2011;31(2):208-13.

25.Wilkinson J, Carryer J, Budge C. Impact of postgraduate education on advanced practice nurse activity - a national survey. International nursing review. 2018;65(3):417-24.

26.Barnhill D, McKillop A, Aspinall C. The impact of postgraduate education on registered nurses working in acute care. Nursing praxis in New Zealand inc. 2012;28(2):27-36.

27.Gijbels H, O'Connell R, Dalton-O'Connor C, O'Donovan M. A systematic review evaluating the impact of post-registration nursing and midwifery education on practice. Nurse education in practice. 2010;10(2):64-9.

28.Ng L, Eley R, Tuckett A. Exploring factors affecting registered nurses' pursuit of postgraduate education in Australia. Nursing \& health sciences. 2016;18(4):435-41.

29.Liao LM, Sun XY, Yu H, Li JW. The association of nurse educational preparation and patient outcomes: Systematic review and meta-analysis. Nurse education today. 2016;42:9-16.

30. Hasnain-Wynia R. Is evidence-based medicine patient-centered and is patient-centered care evidencebased? Health Serv Res. 2006;41(1):1-8.

31.Wagner EH, Bennett SM, Austin BT, Greene SM, Schaefer JK, Vonkorff M. Finding common ground: patient-centeredness and evidence-based chronic illness care. Journal of alternative and complementary medicine (New York, NY). 2005;11 Suppl 1:S7-15.

32.Brouwers MC, Kho ME, Browman GP, Burgers JS, Cluzeau F, Feder G, et al. AGREE II: advancing guideline development, reporting and evaluation in health care. Canadian Medical Association Journal. 2010;182(18):E839-E42.

33.Armstrong MJ, Mullins CD, Gronseth GS, Gagliardi AR. Impact of patient involvement on clinical practice guideline development: a parallel group study. Implementation Science. 2018;13(1):55.

34.Vantomme O, Peremans L. Hoe haalbaar is een verpleegkundige in een niet-forfaitaire groepspraktijk? Huisarts Nu. 2018;47(5):200-5.

35.Supper I, Catala O, Lustman M, Chemla C, Bourgueil Y, Letrilliart L. Interprofessional collaboration in primary health care: a review of facilitators and barriers perceived by involved actors. Journal of public health (Oxford, England). 2015;37(4):716-27.

\section{Tables}


Table 1: Training modules within postgraduate course for practice nurses at the University of Antwerp.

1. Practice organization and communication

- Reception and administrative tasks

- Care planning in the context of a general practice

- Organizing extramural care

- Computer skills

- Knowledge of e-Health

- Interprofessional collaboration

2. Health, prevention and patient education

- Prevention

- Health promotion

- Patient education

- Motivational conversations

3. Target specific protocol care, care planning and case management

- Protocol care and case management

- Guarding the quality of care - evidence based nursing, guidelines and procedures

- Medical technical and nursing technical interventions

- Medical content-related tasks

- Triage

- Systematic screening

- Interprofessional collaboration

4. Clinical practice

Table 1 presents the four training modules within the postgraduate course 'Nursing in a general practice.' at the University of Antwerp. 
Table 2: Socio-demographic and work characteristics.

General practitioner (GP) socio-demographic and work characteristics. $(\mathrm{N}=10)$

\begin{tabular}{|c|c|c|}
\hline \multicolumn{2}{|l|}{ Characteristic } & $\mathrm{n}$ \\
\hline Sex & $\begin{array}{l}\text { Male } \\
\text { Female }\end{array}$ & $\begin{array}{l}5 \\
5\end{array}$ \\
\hline Age (in years) & $\begin{array}{l}\text { Mean (SD) } \\
\text { Min-max }\end{array}$ & $\begin{array}{l}49.4 \\
31-67\end{array}$ \\
\hline Seniority (in years) & $\begin{array}{l}\text { Mean (SD) } \\
\text { Min-max }\end{array}$ & $\begin{array}{l}21.3 \\
2-39\end{array}$ \\
\hline $\begin{array}{l}\text { Experience with practice nurse (PN) support (type of } \\
\text { employment) }\end{array}$ & $\begin{array}{l}\text { PN employee and intern } \\
\text { PN intern }\end{array}$ & $\begin{array}{l}5 \\
5\end{array}$ \\
\hline Years of experience with PN employee & $\begin{array}{l}\text { Mean } \\
\text { Min-max }\end{array}$ & $\begin{array}{l}5.2 \\
2-10\end{array}$ \\
\hline GP colleagues & $\begin{array}{l}\text { Mean } \\
\text { Min-max }\end{array}$ & $\begin{array}{l}3.6 \\
0-5\end{array}$ \\
\hline Other support in the practice & $\begin{array}{l}\text { GP trainees } \\
\text { Practice nurses (in paid } \\
\text { employment) } \\
\text { Administrative assistant } \\
\text { Dietician } \\
\text { Logo } \\
\text { Physiotherapist } \\
\text { Social worker } \\
\text { Psychologist } \\
\text { Diabetes educator } \\
\text { Other support } \\
\end{array}$ & $\begin{array}{l}9 \\
5 \\
8 \\
4 \\
3 \\
2 \\
0 \\
4 \\
1 \\
5 \\
\end{array}$ \\
\hline Location of the practice & $\begin{array}{l}\text { Rural area } \\
\text { City }\end{array}$ & $\begin{array}{l}6 \\
4\end{array}$ \\
\hline Financing of the practice & $\begin{array}{l}\text { Pay for performance } \\
\text { Bundled payment }\end{array}$ & $\begin{array}{l}9 \\
1\end{array}$ \\
\hline
\end{tabular}

\begin{tabular}{|c|c|c|c|c|}
\hline \multicolumn{5}{|c|}{ Practice nurse (PN) socio-demographic and work characteristics. (N=20) } \\
\hline Characteristic & $\begin{array}{c}\text { Total }(\mathrm{N}=20) \\
\mathrm{n}\end{array}$ & $\begin{array}{c}\text { FG1 }(\mathrm{N}=10) \\
\mathrm{n}\end{array}$ & $\begin{array}{c}\mathrm{FG} 2(\mathrm{~N}=4) \\
\mathrm{n}\end{array}$ & $\begin{array}{c}\text { FG3 } \\
(\mathrm{N}=6) \\
\mathrm{n}\end{array}$ \\
\hline $\begin{array}{l}\text { Sex } \\
\text { Female }\end{array}$ & 20 & 10 & 4 & 6 \\
\hline $\begin{array}{l}\text { Age (in years) } \\
\text { Age categories (in years) } \\
20-30 \\
31-40 \\
41-50 \\
51-60\end{array}$ & $\begin{array}{c}\text { Mean (range) } \\
37(22-56) \\
\\
6 \\
7 \\
4 \\
3 \\
\end{array}$ & $\begin{array}{l}3 \\
3 \\
1 \\
3\end{array}$ & $\begin{array}{l}0 \\
3 \\
1 \\
0\end{array}$ & $\begin{array}{l}3 \\
1 \\
2 \\
0\end{array}$ \\
\hline $\begin{array}{l}\text { Seniority } \\
1-3 \text { months } \\
4-12 \text { months } \\
>1 \text { year }\end{array}$ & $\begin{array}{c}11 \\
6 \\
3\end{array}$ & $\begin{array}{c}8 \\
1 \\
1(9.5 \\
\text { years })\end{array}$ & $\begin{array}{c}1 \\
1 \\
2(1.5 \text { and } 6.5 \\
\text { years })\end{array}$ & $\begin{array}{l}2 \\
4 \\
-\end{array}$ \\
\hline $\begin{array}{l}\text { Current type of employment } \\
\text { Employee }\end{array}$ & 4 & 0 & 4 & 0 \\
\hline
\end{tabular}




\begin{tabular}{|c|c|c|c|c|}
\hline $\begin{array}{l}\text { Intern } \\
\text { Both employee and intern }\end{array}$ & $\begin{array}{c}12 \\
4\end{array}$ & $\begin{array}{l}8 \\
2\end{array}$ & $\begin{array}{l}0 \\
0\end{array}$ & $\begin{array}{l}4 \\
2\end{array}$ \\
\hline $\begin{array}{l}\text { Working hours per week } \\
<10 \text { hours } \\
10-20 \text { hours } \\
>20 \text { hours }\end{array}$ & $\begin{array}{l}- \\
- \\
-\end{array}$ & $\begin{array}{l}- \\
- \\
-\end{array}$ & $\begin{array}{l}2 \\
1 \\
1\end{array}$ & $\begin{array}{l}- \\
4 \\
2\end{array}$ \\
\hline $\begin{array}{l}\text { Location of the practice } \\
\text { Rural area } \\
\text { City }\end{array}$ & - & - & $\begin{array}{l}3 \\
1\end{array}$ & $\begin{array}{l}3 \\
3\end{array}$ \\
\hline $\begin{array}{l}\text { Financing of the practice } \\
\text { Pay for performance } \\
\text { Bundled payment }\end{array}$ & - & - & $\begin{array}{l}2 \\
2\end{array}$ & $\begin{array}{l}4 \\
2\end{array}$ \\
\hline
\end{tabular}

Table 3: Discussed topics with practice nurses and GPs.

\begin{tabular}{|c|c|}
\hline Practice nurse interview topics & GP interview topics \\
\hline ICE-breaker & ICE-breaker \\
\hline $\begin{array}{l}\text { - Can you describe the practice you currently work } \\
\text { in? } \\
\text {-Which tasks do you perform in this practice? }\end{array}$ & $\begin{array}{l}\text { - Can you describe your general practice? } \\
\text { - Which tasks do you delegate to practice nurses? }\end{array}$ \\
\hline Non-threatening exploratory questions & Non-threatening exploratory questions \\
\hline $\begin{array}{l}\text { - Questions about collaboration with other health } \\
\text { care professionals, formal/informal consultation } \\
\text { moments, interprofessional patient care. }\end{array}$ & $\begin{array}{l}\text { - Questions about formal/informal consultation } \\
\text { moments, interprofessional patient care, hierarchy. }\end{array}$ \\
\hline Key-questions & Key-questions \\
\hline $\begin{array}{l}\text { - Questions about experienced barriers/facilitators } \\
\text { for interprofessional collaboration, responsibilities } \\
\text { concerning interprofessional collaboration, } \\
\text { importance of education, future needs concerning } \\
\text { interprofessional collaboration in primary care and } \\
\text { education. }\end{array}$ & $\begin{array}{l}\text { - Questions about knowledge of the practice nurses' } \\
\text { expertise, trust in the nurses' competencies, } \\
\text { barriers/facilitators for interprofessional } \\
\text { collaboration, importance of education, future needs } \\
\text { concerning interprofessional collaboration in primary } \\
\text { care and education. }\end{array}$ \\
\hline Closing-questions & Closing-questions \\
\hline $\begin{array}{l}\text { - In an ideal world, what would interprofessional } \\
\text { collaboration in a general practice look like for you? } \\
\text { - Additional comments or questions? }\end{array}$ & $\begin{array}{l}\text { - In an ideal world, how would practice nurses be } \\
\text { integrated in general practices? Where is still room for } \\
\text { improvement? } \\
\text { - Additional comments or questions? }\end{array}$ \\
\hline
\end{tabular}

Table 3 presents an overview of the different topics discussed during the focus group interviews with practice nurses and GPs.

\section{Supplementary Files}

This is a list of supplementary files associated with this preprint. Click to download. 
- supplement1.pdf

Page 24/24 